\title{
A Petri Net View of Mobility
}

\author{
Charles A. Lakos \\ School of Computer Science, University of Adelaide, \\ Adelaide, SA 5005, Australia \\ Charles.Lakos@adelaide.edu.au
}

\begin{abstract}
Mobile systems explore the interplay between locality and connectivity. A subsystem may have a connection to a remote subsystem and use this for communication. Alternatively, it may be necessary or desirable to move the subsystem close to the other in order to communicate. This paper presents a Petri Net formalisation of mobile systems so as to harness the intuitive graphical representation of Petri Nets and the long history of associated analysis techniques.

The proposed formalism starts with modular Petri Nets, where a net is divided into modules which can interact via place and transition fusion. The first change is that the flat module structure is replaced by fully nested modules, called $l o$ cations. The nesting of locations provides a notion of locality while their fusion context determines their connectivity. The transitions constituting a location are constrained so that we can determine when a location is occupied by a subsystem, and when the subsystem shifts from one location to another.

The colourless version of the formalism is conceptually simpler, while the coloured version caters for more dynamic configurations and helps identify isolated subsystems for which garbage collection may be appropriate.
\end{abstract}

\section{Introduction}

There has been considerable interest in mobility and mobile agent systems. Here, components or subsystems migrate from one location to another. When present at a location, the subsystem can interact more readily with co-located subsystems, and less readily (if at all) with remote subsystems. In formalising mobile systems, it is important to be able to evaluate their correctness, the security implications, and the efficiency gains of moving subsystems to avoid latency costs of complex communication protocols.

Mobile systems expose the interplay between locality and connectivity [17]. Connectivity allows one subsystem to interact with another by virtue of having a connection or reference. In a distributed environment, the access from one subsystem to another via a reference is constrained by locality or proximity. It is desirable to capture mobility in a Petri Net formalism, so as to build on the intuitive graphical representation of Petri Nets, the long history of associated analysis techniques [18 19], and to be able to reason about causality and concurrency [6]. However, a simple and general (even elegant) formulation has proved elusive. This paper is an attempt to address this deficiency.

In our presentation, we consider that an appropriate point of departure is that of Modular Petri Nets [3] . Here, a net is made up of a number of subnets, which interact

\footnotetext{
* Supported by an Australian Research Council (ARC) Discovery Grant DP0210524.
} 
in the standard Petri Net way by place and transition fusion. (This makes it possible to model both asynchronous and synchronous interaction, respectively.) It is common to distinguish between nets and systems, where nets capture the structure, and where systems augment the net with the state or marking. We find it convenient to distinguish between subnets, locations and subsystems. A subnet is a Petri Net that captures the structure of a module. It can be considered as a type or class for a set of instances. A location is a subnet in a particular fusion environment. In other words, a location can be considered to be a type with a range of possible interaction capabilities. Finally, a subsystem is a location with a non-empty marking. In other words, it represents an instance of a subnet with a range of interaction capabilities.

Thus, we first extend modular nets with the notion of locations, which are subnets with a specific fusion environment. Locations are nested and thus capture the notion of locality or proximity. The fusion environment determines the connectivity or interaction possibilities for a subsystem. Secondly, modular nets are extended with the capability of shifting locations, which is represented by having transitions with arcs incident on locations. In other words, a subsystem resident in one location can be shifted to another location by firing such a transition. This implies that it is possible to manipulate the marking of a location as a unit.

By analogy with Petri Net theory, it is convenient to distinguish colourless and coloured varieties of mobile systems, which are respectively based on colourless and coloured versions of modular nets [3]4]. In the colourless version, each location may have at most one resident subsystem - the so-called unfolded representation. In the coloured version, multiple subsystems can be resident at a given location, but then the place markings will need to distinguish which tokens belong to which subsystem. This is a so-called folded representation - it requires that each token is associated with a subsystem by including a data value called an identifier.

The paper is organised as follows: Section 2 presents a motivating example of a simple mail agent system. Section 3 presents the formal definitions for a colourless version of mobile systems, while Section 4 presents a coloured version. Section 5 considers possible variations of the formalism and compares it with other approaches in the literature. Section 6 presents the conclusions and avenues for further work.

\section{Example}

We informally introduce the proposed concepts by considering a simple mail agent (shown in Fig.1) and its associated system (shown in Fig. 2). A Petri Net (as in Fig. 1) consists of places, transitions and arcs, together with annotations of these. The places are depicted as ovals and can hold tokens, which then determine the state of the system. The transitions are depicted as rectangles, and determine the possible changes of state. The arcs indicate how the transitions affect the places. In a colourless net, each place holds a number of undifferentiated tokens, and the arcs indicate how many tokens are consumed or generated. So, if an arc from a place to a transition is annotated by the number 2, then that transition can occur only if two tokens in the place can be consumed.

In the example of Fig. 1, there are three places and six transitions. The initial marking shows that place empty is marked while the places has 1 and has 2 are not. In this 


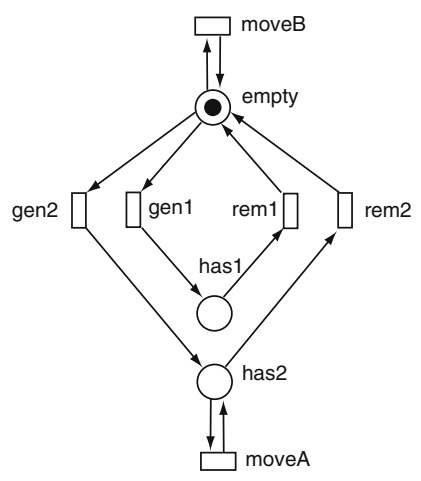

Fig. 1. Subnet for the Agent processes

state, either transition gen 1 or gen 2 can fire - so far, the choice is non-deterministic. This subnet is meant to represent a simple mail agent. With place empty marked, the agent has no mail to deliver, while if place has 1 or has 2 is marked, then the agent has mail to deliver to site 1 or site 2, respectively. The transitions genl and gen 2 generate these mail messages, while transitions rem 1 and rem 2 consume (or deliver) them. Transitions moveA and moveB are used to constrain or allow movement of the agent.

The composite mail system is shown in Fig. 2 Each rounded rectangle is a location, which is a subnet together with a fusion environment. The main or root location is labelled System, and it contains three locations labelled Site0, Site1 and Site2. Within each of these locations is a nested location for the mail agent, labelled LocO, Locl and Loc2, respectively. This example uses transition fusion but not place fusion, and this fusion is indicated in one of three ways - by name correspondence, by a double line, or by a line through the transition (which is used to indicate that it is blocked in this location, i.e. fused to a disabled transition in the environment, which is then not shown to avoid clutter).

At $\mathrm{Site} 0$, the agent resides in location LocO. Its transitions gen 1 and gen 2 can occur, while the others are blocked. These are fused (as indicated by name correspondence) with transitions gen 1 and gen 2 of SiteO. Thus, at SiteO it is possible to generate a message destined for Site1 or Site2, in which case the corresponding place has1 or has 2 of the agent will become marked. Once this has occurred, transition mov01 is enabled. As indicated by the double lines, this transition is fused with the transition mov01 in the location System, which is also fused with the transition mov01 in location Site 1. The transition movO1 in location SiteO has a broad input arc incident on location $\mathrm{LocO}$, while transition mov01 in location Site 1 has a broad output arc incident on location Loc1. This is shorthand for shifting the location of a subsystem - the broad input arc removes all the tokens from the source location, and the broad output arc deposits the tokens into the target location. We comment further on this below.

At Site 1, the agent resides in location Loc1. Its transitions rem 1, moveA and moveB can occur, while the others are blocked. Transition reml in the agent is fused with the similarly-named transition in Site1. This transition allows Sitel to accept a message destined for here. The transition moveA/B in Sitel is fused with either transition moveA 


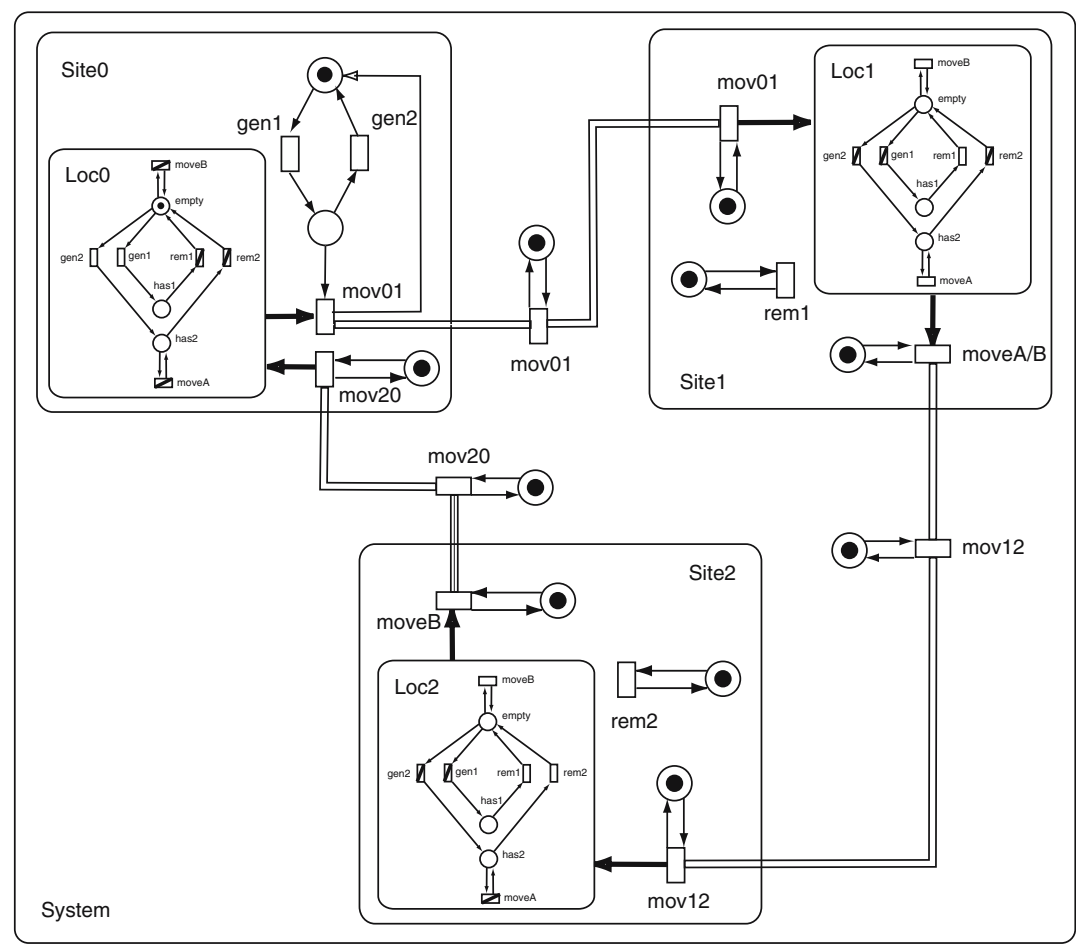

Fig. 2. Composite mail system

or moveB of the agent. This, in turn, is fused with the transition mov 12 of the root instance, and a similarly named transition at Site 2 . Site 2 has a similar structure, and transition rem 2 causes the message destined for this site to be removed.

A location is occupied if at least one of the local places is marked. Thus, in our example, the initial marking indicates that locations System, SiteO, Site1, Site 2 and LocO are occupied, while locations Loc1 and Loc2 are not. In order to ensure that transitions are only enabled for occupied locations, we insist that such transitions have at least one input and one output arc incident on a local (as opposed to a fused) place. Hence, all the side conditions shown in the figure - a side condition is a place with both an input and an output arc incident on an adjacent transition.

The transitions like mov01 which shift the location of a subsystem (or more generally consume a subsystem at a location or generate a subsystem at a location) are shown with a broad arc. This is a shorthand notation for indicating that all the tokens in the local places are consumed. Where a consume is paired with a corresponding generate, it is assumed that the marking is shifted from one location to another. This description is informal, and the notation is syntactic sugar. It is made precise by identifying vacate and occupy transitions. Vacate transitions (if enabled) will have the effect of clearing the marking of its location, i.e. they have input arcs but no output arcs. Dually, occupy transitions (if enabled) will have the effect of setting the marking of its location, i.e. they have output arcs but no input arcs. It is expected that such vacate and occupy tran- 
sitions will normally be fused with environment transitions, and that a vacate transition fused directly or indirectly with an occupy transition will have the effect of shifting the subsystem. The broad arcs incident on a location are thus syntactic sugar for a number of such vacate and occupy transitions - one for every possible marking of the location. This has theoretical implications which we consider in Section 3 .

It is worth noting that for Site 0 , the shifting of the agent is determined solely by the site, whereas for Site 1 and Site 2 the agent collaborates with the shift transition.

\section{Modular Petri Nets for Mobility}

In this section we present a definition of mobile systems in terms of Modular Petri Nets. In Section 4 we present a definition in terms of Modular Coloured Petri Nets.

Definition 1 (Multiset). A multiset over set $S$ is a mapping $m: S \rightarrow \mathbb{N}$, where $m(s)$ gives the number of occurrences of $s$. The set of all multisets over set $S$ is denoted $\mu(S)$.

Definition 2 (Petri Net). A Petri Net $(P N)$ is a tuple $P N=(P, T, W)$ where:

1. $P$ is a finite set of places.

2. $T$ is a finite set of transitions with $P \cap T=\emptyset$.

3. $W:(P \times T) \cup(T \times P) \rightarrow \mathbb{N}$ is an arc weight function.

The arc weight function $W$ indicates the number of tokens consumed or generated by the firing of the transition. If an arc weight is zero, the arc is not drawn.

Definition 3 (PN Markings and Steps). For a Petri Net PN, a marking is a mapping $M: P \rightarrow \mathbb{N}$, i.e. $M \in \mu(P)$, and $a$ step is a mapping $Y: T \rightarrow \mathbb{N}$, i.e. $Y \in \mu(T)$. A Petri Net System is a Petri Net together with an initial marking.

The example of Fig, 1 is a Petri Net System where the arcs shown have a weight of 1 and the arcs which have not been drawn have a weight of 0 . Further, the initial marking of place empty is one token, while other places hold no tokens.

Definition 4 (PN Behaviour). For a Petri Net PN, a step $Y$ is enabled in marking $M$, denoted $M[Y\rangle$, if $\forall p \in P: \Sigma_{t \in Y} W(p, t) \leq M(p)$. If step $Y$ is enabled in marking $M$, then it may occur, leading to marking $M^{\prime}$, denoted $M[Y\rangle M^{\prime}$, where $\forall p \in P: M^{\prime}(p)=$ $M(p)-\Sigma_{t \in Y} W(p, t)+\Sigma_{t \in Y} W(t, p)$. We write $[M\rangle$ for the markings reachable from $M$ by the occurrence of zero or more steps.

The above definitions are quite conventional. They capture the requirement that a place must have sufficient tokens to satisfy all consume demands of the step, and that when the step occurs, a place receives tokens from all generate actions. We now depart from convention by defining locations and mobile systems. These have been motivated by Modular Petri Nets [4], but here we retain the nested structure of the modules, which we call locations, and thereby capture the notion of locality.

Definition 5 (PN Location). A Petri Net Location is a tuple $L=\left(S_{L}, P_{L}, T_{L}, W_{L}\right)$ where: 
1. $S_{L}$ is a finite set of locations. We define $\operatorname{loc}(L)=\bigcup_{s \in S_{L}} \operatorname{loc}(s) \cup\{L\}$. We require $\forall s \in S_{L}: \operatorname{loc}(s) \cap\{L\}=\emptyset$.

2. $\left(P_{L}, T_{L}, W_{L}\right)$ is a Petri Net. We define plc $(L)=\bigcup_{s \in S_{L}} p l c(s) \cup\left\{P_{L}\right\}$ and $\operatorname{trn}(L)=\bigcup_{s \in S_{L}} \operatorname{trn}(s) \cup\left\{T_{L}\right\}$.

Thus, locations are properly nested nets. Locations are unique, as are places and transitions (which are differentiated by the location in which they reside). We can define markings, steps and behaviour for individual locations just as for Petri Nets, but we defer such definitions to mobile systems.

Definition 6 (Mobile System). A Mobile System is a tuple $M S=\left(L_{0}, P F, T F, M_{0}\right)$ where:

1. $L_{0}$ is a location, called the root location. We define $P=\operatorname{plc}\left(L_{0}\right)$ and $T=\operatorname{trn}\left(L_{0}\right)$.

2. $P F$ is a set of place fusion sets where $\bigcup_{p f \in P F} p f=P$ and $\forall p f_{1}, p f_{2} \in P F$ : $p f_{1} \cap p f_{2} \neq \emptyset \Rightarrow p f_{1}=p f_{2}$.

3. $T F$ is a set of transition fusion sets where $\bigcup_{t f \in T F} t f=T$ and $\forall t f_{1}, t f_{2} \in T F$ : $t f_{1} \cap t f_{2} \neq \emptyset \Rightarrow\left|t f_{1}\right|=\left|t f_{2}\right|$.

4. $M_{0}$ is the initial marking of the location.

The set of place fusion sets covers all places and the fusion sets cannot partially overlap. This is in contrast to the definition of Modular Petri Nets [4], where the transitive closure of the fusion sets is used to determine the equivalence classes over places, which are then called place instance groups. Our approach means that each place fusion set corresponds to one place instance group. Similarly, the set of transition fusion sets is required to cover all transitions and if one transition occurs in more than one transition fusion set then these sets must have the same size. Again, this is more restricted than that of Modular Petri Nets, but it is not a theoretical restriction given that every transition can be duplicated so there is one duplicate per occurrence in a fusion set.

Definition 7 (MS Markings and Steps). For mobile system MS, a marking is a mapping $M: P \rightarrow \mathbb{N}$, where $\forall p f \in P F: \forall p_{1}, p_{2} \in p f: M\left(p_{1}\right)=M\left(p_{2}\right)=M(p f)$, and a step is a mapping $Y: T \rightarrow \mathbb{N}$, where $\forall t f \in T F: \forall t_{1}, t_{2} \in t f: Y(t f)=Y\left(t_{1}\right)=$ $Y\left(t_{2}\right)$.

In a mobile system, as in modular nets, the markings of fused places are identical, and the multiplicity of fused transitions in a step are identical. This justifies defining the markings and steps of such a system in terms of place fusion sets and transition fusion sets. It is then appropriate to extend the definition of the arc weight function $W$ to apply to place fusion sets and transition fusion sets, i.e.

$$
\forall\left(f_{1}, f_{2}\right) \in(P F \times T F) \cup(T F \times P F): W\left(f_{1}, f_{2}\right)=\Sigma_{x \in f_{1}, y \in f_{2}} W(x, y)
$$

Definition 8 (MS Behaviour). For a mobile system MS, a step $Y$ is enabled in marking $M$ if $\forall p f \in P F: \Sigma_{t f \in Y} W(p f, t f) \leq M(p f)$. If step $Y$ is enabled in marking $M$, then it may occur, leading to marking $M^{\prime}$ where $\forall p f \in P F: M^{\prime}(p f)=M(p f)-$ $\Sigma_{t f \in Y} W(p f, t f)+\Sigma_{t f \in Y} W(t f, p f)$. 
Definition 9. For a Mobile System MS we classify places and transitions as follows:

1. $L P=\{p \in P \mid \exists p f \in P F: p f=\{p\}\}$ is the set of local places.

2. $E P=P-L P$ is the set of exported places.

3. $L T=\{t \in T \mid \exists t f \in T F: t f=\{t\}\}$ is the set of local transitions.

4. $E T=T-L T$ is the set of exported transitions.

5. $V T=\{t \in T \mid \exists p \in L P: W(p, t)>0 \wedge \forall p \in P: W(t, p)=0\}$ is the set of vacate transitions.

6. $O T=\{t \in T \mid \exists p \in L P: W(t, p)>0 \wedge \forall p \in P: W(p, t)=0\}$ is the set of occupy transitions.

7. $R T=\left\{t \in T \mid \exists p_{1}, p_{2} \in L P: W\left(t, p_{1}\right)>0 \wedge W\left(p_{2}, t\right)>0\right\}$ is the set of regular transitions.

We distinguish local as opposed to exported places and transitions - exported entities are fused to at least one other. With the notion of mobility, we are interested in whether a transition interacts with local places, since this determines if the location is occupied. Accordingly, we classify transitions by their interaction with local places.

Definition 10 (Well-formed). A Mobile System MS is well-formed if:

1. All transitions are vacate, occupy or regular transitions, i.e. $T=V T \cup O T \cup R T$.

2. Vacate transitions empty a location for all reachable markings, i.e. $\forall L \in \operatorname{loc}\left(L_{0}\right)$ : $\forall t \in V T \cap T_{L}: \forall M \in\left[M_{0}\right\rangle: M[t\rangle M^{\prime} \Rightarrow \forall p \in L P \cap p l c(L): M^{\prime}(p)=\emptyset$.

3. Occupy transitions fill a location for all reachable markings, i.e. $\forall L \in \operatorname{loc}\left(L_{0}\right)$ : $\forall t \in O T \cap T_{L}: \forall M \in\left[M_{0}\right\rangle: M[t\rangle M^{\prime} \Rightarrow \forall p \in L P \cap p l c(L): M(p)=\emptyset$.

The above definition of a well-formed mobile system is the key definition that supports mobility. We identify a location as being occupied if a local place is marked. A vacate transition has the effect of transforming an occupied location (and its nested locations) to unoccupied, while an occupy transition has the effect of transforming an unoccupied location (and its nested locations) to occupied. A regular transition has the effect of ensuring that an occupied location stays occupied.

The requirement that all transitions fall into one of these three categories ensures that occupy transitions are the only ones that can become enabled if the location to which they belong is unoccupied. The requirements that vacate transitions make a location unoccupied and that occupy transitions make a location occupied apply to all reachable markings. It is therefore debatable whether these should be classified as requirements for being well-formed or well-behaved. Our choice of terminology reflects our intention that these conditions should be determined from the structure of the net, without the need for reachability analysis. Essentially, the problem is one of incorporating clear and set arcs [12] — the nature of these arcs determines whether reachability and boundedness are decidable for this form of Petri Net [7]. If all places in all locations are bounded, then it will be possible to incorporate complementary places and there will be a finite number of possibilities for the clear and set arcs, and hence for clearing and setting the marking of the location. If there are unbounded places, then we will need the generalised form of clear and set arcs [12] which make reachability and boundedness undecidable [7]. 
We refer to a location together with a non-empty marking as a subsystem. By incorporating vacate and occupy transitions, the above formalism is sufficient for studying mobility - fusing a vacate transition in one location with an occupy transition in another corresponds to shifting the location of a subsystem. For notational convenience, we introduce the broad arcs incident on locations, as in Section 2 . These are a shorthand for sets of vacate and/or occupy transitions - one for each possible reachable marking of the location. They summarise the possibility of shifting the location of a subsystem whatever its current state. Again, with bounded places, the possible alternatives can be enumerated. With unbounded places, the generalised clear and set arcs are required.

Definition 11 (Isolated subsystem). Given a Mobile System MS in marking M, a transition sequence $t_{1} t_{2} \ldots t_{n}$ is a causal sequence if there are markings $M_{1}, M_{2}, \ldots M_{n}$ such that $M\left[t_{1}\right\rangle M_{1}\left[t_{2}\right\rangle M_{2} \ldots\left[t_{n}\right\rangle M_{n}$ and $\forall k \in 1 . .(n-1): \exists p \in P: W\left(t_{k}, p\right)>$ $0 \wedge W\left(p, t_{k+1}\right)>0$. Given a Mobile System $M S$, a subsystem resident in location $L$ is isolated in marking $M$ if there is no causal sequence $t_{1} t_{2} \ldots t_{n}$ with $t_{1} \in T_{L}$ and $t_{n} \in T_{L_{0}}$.

The definition of a causal sequence is derived from causal nets [5]. It captures the notion of causal dependency between transitions $t_{1}$ and $t_{n}$, since the output of each transition is consumed by the subsequent transition. We then say that the subsystem in location $L$ is isolated if there is no such causal sequence with $t_{1}$ in location $L$ and $t_{n}$ in the root location. In other words, the firing of transitions of the subsystem in location $L$ cannot affect, directly or indirectly, the root location.

If we are only interested in studying the behaviour of the root location, we can reduce the size of the state space by eliminating isolated subsystems. For the moment, this observation seems somewhat pointless because we need the reachability graph to determine whether a location is isolated. We revisit this issue in the coloured version of mobile systems.

\section{Modular Coloured Petri Nets for Mobility}

We now extend the definition of mobile systems to incorporate colour, i.e. data values. One of the benefits of doing so — just as for coloured nets — is to have a more concise notation. It will now be possible to have multiple subsystems resident in the one location, provided that the elements of the subsystems are differentiated by the use of identifiers, a special kind of data value from a set $I D$. Local places will have tokens tagged by the subsystem identifier, and local transitions will have firing modes similarly tagged. Fused places will have tokens tagged by the identifiers of all subsystems with one of the fused places, and similarly, fused transitions will have firing modes tagged by identifiers of all participating subsystems. Thus, we dictate that tokens and transition firing modes have colour sets which are tuples, the first element of which is the multiset of identifiers which determine the subsystem(s) to which they belong. We use a projection function $\pi_{1}$ to select the first element of such tuples. The constraints on the consistent use of identifiers are found in Definition 20.

Definition 12 (Coloured Petri Net). A Coloured Petri Net $(C P N)$ is a tuple CPN= $(\Sigma, P, T, \theta, W)$ where: 
1. $\Sigma$ is a set of colour sets (or types).

2. $P$ is a finite set of places.

3. $T$ is a finite set of transitions with $P \cap T=\emptyset$.

4. $\theta: P \cup T \rightarrow \Sigma$ is a colour function giving the colour set associated with each place and transition.

5. $W:(P \times T) \cup(T \times P) \rightarrow \Sigma \rightarrow \Sigma$ is an arc weight function where $W(p, t), W(t, p) \in \mu(\theta(t)) \rightarrow \mu(\theta(p))$.

The definition of CPNs is simplified to that of [3]. As in Definition 2, we combine the specification of arcs and their inscriptions into one entity, namely the arc weight function $W$. We use $\theta$ to specify the colour set associated with each place. Symmetrically, we also use $\theta$ to specify the colour set associated with each transition - the values of this colour set determine the allowable firing modes of the transition, thus making it superfluous to specify a transition guard.

Definition 13 (CPN Markings and Steps). For a Coloured Petri Net a marking is a mapping $M: P \rightarrow \Sigma$, where $M(p) \in \mu(\theta(p))$, and a step is a mapping $Y: T \rightarrow \Sigma$, where $Y(t) \in \mu(\theta(t))$. A Coloured Petri Net System is a Coloured Petri Net together with an initial marking.

Definition 14 (CPN Behaviour). For a Coloured Petri Net CPN, a step $Y$ is enabled in marking $M$ if $\forall p \in P: \Sigma_{(t, c) \in Y} W(p, t)(c) \leq M(p)$. If step $Y$ is enabled in marking $M$, then it may occur, leading to marking $M^{\prime}$ where $\forall p \in P: M^{\prime}(p)=$ $M(p)-\Sigma_{(t, c) \in Y} W(p, t)(c)+\Sigma_{(t, c) \in Y} W(t, p)(c)$.

Given our use of transition firing modes, the elements of a step are transition-mode pairs, rather than transition-binding pairs, as in [3]. Otherwise, the above definitions are quite conventional.

Definition 15 (Coloured Location). A Coloured Location is a tuple $C L=\left(S_{C L}, \Sigma_{C L}, P_{C L}, T_{C L}, \theta_{C L}, W_{C L}\right)$ where:

1. $S_{C L}$ is a finite set of locations. We define loc $(C L)=\bigcup_{s \in S_{C L}} \operatorname{loc}(s) \cup\{C L\}$.

2. $\left(\Sigma_{C L}, P_{C L}, T_{C L}, \theta_{C L}, W_{C L}\right)$ is a Coloured Petri Net. We define $p l c(C L)=\bigcup_{s \in S_{C L}} p l c(s) \cup\left\{P_{C L}\right\}$ and $\operatorname{trn}(C L)=\bigcup_{s \in S_{C L}} \operatorname{trn}(s) \cup\left\{T_{C L}\right\}$.

Coloured locations, like colourless locations of Definition 5, are modules which retain the nesting structure. As before, we can define markings, steps and behaviour for individual coloured locations, but we defer such definitions to coloured mobile systems.

Definition 16 (Coloured Mobile System). A Coloured Mobile System is a tuple $C M S=\left(C L_{0}, P F, T F, M_{0}\right)$ where:

1. $C L_{0}$ is a coloured location, called the root location. We define $P=p l c\left(C L_{0}\right)$ and $T=\operatorname{trn}\left(C L_{0}\right)$.

2. $P F$ is a set of place fusion sets where $\bigcup_{p f \in P F} p f=P$ and $\forall p f_{1}, p f_{2} \in P F$ : $p f_{1} \cap p f_{2} \neq \emptyset \Rightarrow p f_{1}=p f_{2}$.

3. $T F$ is a set of transition fusion sets where $\bigcup_{t f \in T F} t f=T$ and $\forall t f_{1}, t f_{2} \in T F$ : $t f_{1} \cap t f_{2} \neq \emptyset \Rightarrow\left|t f_{1}\right|=\left|t f_{2}\right|$.

4. $M_{0}$ is the initial marking of the location. 
As in Definition 6 the set of place fusion sets covers all places and the fusion sets do not partially overlap. Thus, each place fusion set corresponds to a place instance group of [3]. Similarly, the set of transition fusion sets covers all transitions and if one transition occurs in more than one fusion set, then the sets must have the same size.

Definition 17 (CMS Markings and Steps). For a Coloured Mobile System CMS, a marking is a mapping $M: P \rightarrow \Sigma$, where $M(p) \in \mu(\theta(p))$ and $\forall p f \in P F: \forall p_{1}, p_{2} \in$ pf : $M\left(p_{1}\right)=M\left(p_{2}\right)=M(p f)$, and a step is a mapping $Y: T \rightarrow \Sigma$, where $Y(t) \in \mu(\theta(t))$ and $\forall t f \in T F: \forall t_{1}, t_{2} \in t f: Y(t f)=Y\left(t_{1}\right)=Y\left(t_{2}\right)$.

In a coloured mobile system, as in coloured modular nets, the markings of fused places are identical, and the multiplicity of fused transitions in a step are identical. As for colourless mobile systems, this justifies defining the markings and steps of such a system in terms of place fusion sets and transition fusion sets. It is also appropriate to extend the definition of the arc weight function $W$ to apply to place fusion sets and transition fusion sets in the same way as for colourless mobile systems.

Definition 18 (CMS Behaviour). For a coloured mobile system CMS, a step $Y$ is enabled in marking $M$ if $\forall p f \in P F: \Sigma_{(t f, c) \in Y} W(p f, t f)(c) \leq M(p f)$. If step $Y$ is enabled in marking $M$, then it may occur, leading to marking $M^{\prime}$ where $\forall p f \in P F$ : $M^{\prime}(p f)=M(p f)-\Sigma_{(t f, c) \in Y} W(p f, t f)(c)+\Sigma_{(t f, c) \in Y} W(t f, p f)(c)$.

Definition 19. For a Coloured Mobile System CMS we define:

1. $L P=\{p \in P \mid \exists p f \in P F: p f=\{p\}\}$ is the set of local places.

2. $E P=P-L P$ is the set of exported places.

3. $L T=\{t \in T \mid \exists t f \in T F: t f=\{t\}\}$ is the set of local transitions.

4. $E T=T-L T$ is the set of exported transitions.

5. $V T=\{t \in T \mid \exists p \in L P: \forall c \in \theta(t): W(p, t)(c)>\emptyset \wedge \forall p \in P: \forall c \in \theta(t)$ : $W(t, p)(c)=\emptyset\}$ is the set of vacate transitions.

6. $O T=\{t \in T \mid \exists p \in L P: \forall c \in \theta(t): W(t, p)(c)>\emptyset \wedge \forall p \in P: \forall c \in \theta(t)$ : $W(p, t)(c)=\emptyset\}$ is the set of occupy transitions.

7. $R T=\left\{t \in T \mid \exists p_{1}, p_{2} \in L P: \forall c \in \theta(t): W\left(t, p_{1}\right)(c)>\emptyset \wedge W\left(p_{2}, t\right)(c)>\emptyset\right\}$ is the set of regular transitions.

As in Definition 9, we distinguish local versus exported places and transitions, and we classify transitions by their interaction with local places.

Definition 20 (Consistent). A Coloured Mobile System CMS is consistent if:

1. The colour set for a place is given by a tuple with the first element being a multiset of identifiers, the size being determined by the size of the relevant fusion set, i.e. $\forall p f \in P F: \forall p \in p f: \theta(p)=\mu(I D) \times \ldots \wedge\left|\pi_{1}(\theta(p))\right|=|p f|$.

2. The colour set for a transition is given by a tuple with the first element being a multiset of identifiers, the size being determined by the size of the relevant fusion set, i.e. $\forall t f \in T F: \forall t \in t f: \theta(t)=\mu(I D) \times \ldots \wedge\left|\pi_{1}(\theta(t))\right|=|t f|$.

3. The firing mode of each transition shares an identifier with the consumed tokens, i.e. $\forall p \in P: \forall t \in T: \forall c \in \theta(t): \forall c^{\prime} \in W(p, t)(c): \pi_{1}(c) \cap \pi_{1}\left(c^{\prime}\right) \neq \emptyset$. 
4. The firing mode of each transition shares an identifier with the generated tokens, i.e. $\forall p \in P: \forall t \in T: \forall c \in \theta(t): \forall c^{\prime} \in W(t, p)(c): \pi_{1}(c) \cap \pi_{1}\left(c^{\prime}\right) \neq \emptyset$.

5. Distinct subsystems have distinct identifiers, i.e. $\forall M \in\left[M_{0}\right\rangle: \forall C L_{1}, C L_{2} \in$ $\operatorname{loc}\left(C L_{0}\right): \forall p_{1} \in L P \cap C L_{1}: \forall p_{2} \in L P \cap C L_{2}: \forall c_{1} \in M\left(p_{1}\right): \forall c_{2} \in$ $M\left(p_{2}\right): C L_{1} \neq C L_{2} \Rightarrow \pi_{1}\left(c_{1}\right) \cap \pi_{2}\left(c_{2}\right)=\emptyset$.

6. The consumed tokens provide all the identifiers found in the transition firing modes, i.e. $\forall t \in T: \forall c_{1}, c_{2} \in \theta(t):\left(\forall p \in P: W(p, t)\left(c_{1}\right)=W(p, t)\left(c_{2}\right)\right) \Rightarrow c_{1}=c_{2}$.

As discussed earlier, we use identifiers from the set $I D \in \Sigma$ to differentiate the multiple subsystems resident in the one location and we require that those identifiers be used consistently. Since tokens in a fused place belong to multiple subsystems, they are identified by all the associated subsystem identifiers. The same applies to fused transitions, and hence points 1 and 2 above. (We use multisets of identifiers so as to handle fusion of places and transitions from the same subsystem.) Furthermore, the firing of a transition should only remove or generate tokens for matching subsystems, and hence points 3 and 4 . Finally, we do not wish to allow transitions to invent identifiers, which may or may not be associated with existing subsystems. Therefore, we require that a transition's firing mode is solely determined by the consumed tokens, which is point 6 above. We now define well-formed nets by extending the constraints on vacate and occupy transitions to cater for coloured nets.

Definition 21 (Well-formed). A Coloured Mobile System CMS is well-formed if:

1. All transitions are vacate, occupy or regular transitions, i.e. $T=V T \cup O T \cup R T$.

2. Vacate transitions empty a location of a subsystem for all reachable markings, i.e. $\forall C L \in \operatorname{loc}\left(C L_{0}\right): \forall t \in V T \cap T_{C L}: \forall M \in\left[M_{0}\right\rangle: M[(t, c)\rangle M^{\prime} \Rightarrow \forall p \in$ $L P \cap p l c(C L): \forall c^{\prime} \in M^{\prime}(p): \pi_{1}(c) \cap \pi_{1}\left(c^{\prime}\right)=\emptyset$.

3. Occupy transitions fill an empty location with a subsystem for all reachable markings, i.e. $\forall C L \in \operatorname{loc}\left(C L_{0}\right): \forall t \in O T \cap T_{C L}: \forall M \in\left[M_{0}\right\rangle: M[(t, c)\rangle M^{\prime} \Rightarrow \forall p \in$ $L P \cap p l c(C L): \forall c^{\prime} \in M(p): \pi_{1}(c) \cap \pi_{1}\left(c^{\prime}\right)=\emptyset$.

Definition 22 (Isolated subsystem). Given a Coloured Mobile System CMS in marking $M$, a transition sequence $\left(t_{1}, c_{1}\right)\left(t_{2}, c_{2}\right) \ldots\left(t_{n}, c_{n}\right)$ is a causal sequence if there are markings $M_{1}, M_{2}, \ldots M_{n}$ such that $M\left[\left(t_{1}, c_{1}\right)\right\rangle M_{1}\left[\left(t_{2}, c_{2}\right)\right\rangle M_{2} \ldots\left[\left(t_{n}, c_{n}\right)\right\rangle M_{n}$ and $\forall k \in 1$... $(n-1): \exists p \in P: W\left(t_{k}, p\right)\left(c_{k}\right) \cap W\left(p, t_{k+1}\right)\left(c_{k+1}\right) \neq \emptyset$. Given a Coloured Mobile System CMS, a subsystem resident in location $C L$ is isolated in marking $M$ if there is no causal sequence $\left(t_{1}, c_{1}\right)\left(t_{2}, c_{2}\right) \ldots\left(t_{n}, c_{n}\right)$ with $t_{1} \in T_{C L}$ and $t_{n} \in T_{C L_{0}}$.

The definition of an isolated subsystem is simply a coloured version of Definition 11. However, with the use of colours and the differentiation of subsystems by identifiers, it is possible to approximate the identification of isolated subsystems. Suppose that a subsystem with identifier $i d$ is resident in location $C L$. Suppose further that there is no place outside $C L$ holding tokens which include the value $i d$. Then the subsystem is isolated and can be eliminated provided we are only interested in the behaviour of the root location.

This follows from the fact that there can only be an appropriate causal sequence if location $C L$ can fire a fused transition or can access tokens from a fused place. In the 
former case, the fusion partner will have to have a firing mode which includes $i d$, which in turn will need to come from a consumed token (points 2, 4, 6 from Definition 20). In the case of fused places, the accessed token will need to have an identifier which includes $i d$ (point 1 of Definition 20). Both of these are excluded if there is no place outside of $C L$ which holds tokens including the value $i d$.

The above is essentially garbage collection for mobile systems. It provides a sufficient condition for a subsystem being isolated, but it is not a necessary condition.

\section{Variations and Related Work}

The above Petri Net formalisms for mobile systems can be varied in a number of ways. As is common with Petri Nets, this formulation appears to be rather static - the number of locations and the communication partners at each location are determined in advance. However, a more dynamic version can be achieved by suitable use of colours or types. Firstly, we could annotate transitions with expressions of the form $v=n e w$ $S$ (arguments) and v.connect(arguments). These would, respectively, generate a new instance of subnet $S$ and move the subsystem identified by $v$, with the arguments in both cases indicating the new fusion context. However, the possible fusion contexts can be enumerated in advance by analysing the syntax of those calls. Secondly, we could use synchronous channels [2] to determine communication partners dynamically — it has been shown that synchronous channels are semantically equivalent to the standard transition fusion that we adopted [2]. Thirdly, we could use colours not just to distinguish multiple subsystems resident in the one location but also to fold multiple locations (with similar fusion contexts) onto one, with colours identifying the different locations.

The formulation above can be interpreted as a variant of Object-Based Petri Nets (OBPNs) where the subnets correspond to classes and the subsystems correspond to instances. An earlier formulation of OBPNs [13], with a view to capturing inheritance, constrained classes to interact either by place fusion or transition fusion. The current proposal allows both. There, explicit destruction of objects was assumed - here we allow for garbage collection. There, catering for multi-level activity was rather complex - here we have a simpler formulation based on Modular Petri Net semantics. A simplifying factor is that shifting the location of a subsystem is not combined with other internal activity, since vacate and occupy transitions cannot, respectively, generate and consume tokens. However, such an extension could be incorporated.

The Nets-within-Nets paradigm proposed by Valk has been the focus of a significant effort in terms of object-oriented design and mobility [8 9 10 20]. The fundamental notion is that there are (at least) two levels of Petri Nets - the system net provides the most abstract view of the system. The tokens resident in the places of the system net may be black tokens (with no associated data) or representations of object nets. The two-level hierarchy can be generalised to an arbitrary number of levels, but that is not necessary for our purposes. Three different semantics have been proposed for the nets-within-nets paradigm - a reference semantics (where tokens in the system net are references to common object nets), a value semantics (where tokens in the system net are distinct object nets), and a history process semantics (where tokens in the system net are object net processes) [20]. The reference semantics (as supported by the Renew tool [11]) 
has been used to model mobile agent systems [8]9]. However, a reference semantics provides a global reference scope, so that connectivity is enhanced but locality becomes meaningless. These authors have acknowledged that a value semantics is really required for mobility [10]. Then, locality is meaningful but connectivity is more constrained an object net residing in a system place can primarily synchronise with the system transitions adjacent to the place. In other words, the object net token has to be removed from the place in order to interact with it. The interaction between co-resident tokens has more recently been added using another form of synchronous channel. However, the notation in Renew suggests that the interaction is achieved by the two object nets being accessed as side conditions of a system net transition.

Our proposal has certain points of contact with the nets-within-nets paradigm. The notation of having arcs incident on locations is akin to system net places containing object nets which can be removed (or added) by adjacent system net transitions. However, our locations have a more general fusion context. We have also refined the results of [10] in noting that if locations have bounded places, then we obviate the need for generalised clear and set arcs for shifting subsystem locations, and hence reachability and boundedness can remain decidable.

There have been a number of calculi proposed for working with mobility. Mobility was one of the key motivations behind the $\pi$-calculus [16]. However, the $\pi$-calculus did not explore the interplay between connectivity and locality — it had a flexible method for exchanging names and thus modifying connectivity, but there was no sense in which the connectivity was limited by locality. (The scope rules simply limited the accessibility to names.)

The ambient calculus [1] identifies ambients as a sphere of computation. They are properly nested which then determines locality. Capabilities are provided for entering, leaving and dissolving ambients. Movement across ambient boundaries can be subjective — the process in the ambient decides to employ the capability — or objective the process outside the ambient dictates the move. As in the $\pi$-calculus, connectivity is provided by the ability to communicate capabilities or names over channels.

The seal calculus [21] identifies seals as agents or mobile computations. Here, seal boundaries are the main protection mechanism and seal communication is restricted to a single level in the hierarchy. Mobility is not under the control of a seal but of its parent - thus subjective moves of the ambient calculus are not supported.

The capabilities of the above calculi can be broadly mapped into the formalisms of this paper which can make it possible to specify and reason about causality and concurrency, as in [6]. Ambients and seals can be mapped to locations. We can cater for synchronous and asynchronous communication. Moves are objective, and fusion can be constrained to the enclosing location as in the Seal calculus.

\section{Conclusions}

This paper has proposed a Petri Net formalism suitable for studying mobility, and specifically the interplay between locality and connectivity. It has extended Modular Petri Nets with the notion of nested modules called locations. The nesting of modules determines locality while the fusion context of each module determines connectivity. 
Locations are constrained so that the firing of their transitions depends on the locations being occupied. Another key extension is the identification of vacate and occupy transitions, which change the occupied status of locations. For notational convenience, we add arcs incident on locations to represent multiple vacate and occupy transitions. We have also identified isolated subsystems which cannot affect, either directly or indirectly, the root location. The analysis of the system could be simplified by removing such isolated subsystems from further consideration. In the coloured version of mobile systems, the question of isolated subsystems can be resolved with techniques similar to garbage collection for object-oriented systems.

The proposed Petri Net formalism for mobility is quite simple and general even though the changes to Modular Petri Nets are not extensive. Consequently, it will be relatively simple to study mobile systems using currently available modular analysis tools such as Maria [15], which already has support for nested modules. With a bit more effort, it is also possible to map these mobile systems into Hierarchical Coloured Petri Nets and analyse them in the tool Design/CPN [14].

Future work will explore front ends to existing tools in order to facilitate the modelling of mobile systems, as well as their analysis. We are also interested in exploring the analysis possibilities for realistic case studies.

\section{Acknowledgements}

The author acknowledges early discussions with Thomas Mailund about state space exploration of Object-Based systems, and the helpful comments of Glenn Lewis.

\section{References}

1. L. Cardelli and A. Gordon. Mobile Ambients. In M. Nivat, editor, Foundations of Software Science and Computational Structures, volume 1998 of Lecture Notes in Computer Science, pages 140-155. Springer Verlag, 1998.

2. S. Christensen and N.D. Hansen. Coloured Petri Nets Extended with Channels for Synchronous Communication. In R. Valette, editor, 15th International Conference on the Application and Theory of Petri Nets, volume 815 of Lecture Notes in Computer Science, pages 159-178, Zaragoza, 1994. Springer-Verlag.

3. S. Christensen and L. Petrucci. Modular State Space Analysis of Coloured Petri Nets. In G. De Michelis and M. Diaz, editors, Application and Theory of Petri Nets, volume 935 of Lecture Notes in Computer Science, pages 201-217. Springer-Verlag, Berlin, 1995.

4. S. Christensen and L. Petrucci. Modular analysis of Petri Nets. The Computer Journal, 43(3):224-242, 2000.

5. J. Desel and W. Reisig. Place/Transition Petri Nets. In W. Reisig and G. Rozenberg, editors, Lectures on Petri Nets I: Basic Models, volume 1491 of Lecture Notes in Computer Science, pages 122-173. Springer, Dagstuhl, 1998.

6. R. Devillers, H. Klaudel, and M. Koutny. Petri Net Semantics of the Finite $\pi$-Calculus. In D.de Frutos-Escrig and M. Nunez, editors, Formal Techniques for Networked and Distributed Systems, volume 3235 of Lecture Notes in Computer Science, pages 309-325, Madrid, 2004. Springer-Verlag. 
7. C. Dufourd, A. Finkel, and Ph. Schnoebelen. Reset nets between decidability and undecidability. In K. Larsen, S. Skyum, and G.Winskel, editors, 25th International Colloquium on the Automata, Languages and Programming, volume 1443 of Lecture Notes in Computer Science, pages 103-115, Aalborg, 1998. Springer-Verlag.

8. M. Köhler, D. Moldt, and H. Rölke. Modelling the Structure and Behaviour of Petri Net Agents. In J.-M. Colom and M. Koutny, editors, International Conference on the Application and Theory of Petri Nets, volume 2075 of Lecture Notes in Computer Science, pages 224241, Newcastle, 2001. Springer-Verlag.

9. M. Köhler, D. Moldt, and H. Rölke. Modelling Mobility and Mobile Agents Using Nets within Nets. In W. van der Aalst and E. Best, editors, International Conference on the Application and Theory of Petri Nets, volume 2679 of Lecture Notes in Computer Science, pages 121-139, Eindhoven, 2003. Springer-Verlag.

10. M. Köhler and H. Rölke. Properties of Object Petri Nets. In J. Cortadella and W. Reisig, editors, International Conference on the Application and Theory of Petri Nets, volume 3099 of Lecture Notes in Computer Science, pages 278-297, Bologna, 2004. Springer-Verlag.

11. O. Kummer, F. Wienberg, M. Duvigneau, J. Schumacher, M. Köhler, D. Moldt, H. Rölke, and R. Valk. An extensible editor and simulation engine for Petri nets: Renew. In J. Cortadella and W. Reisig, editors, 25th International Conference on Application and Theory of Petri Nets (ICATPN 2004), volume 3099 of Lecture Notes in Computer Science, pages 484-493, Bologna, Italy, 2004. Springer.

12. C. Lakos and S. Christensen. A General Systematic Approach to Arc Extensions for Coloured Petri Nets. In R. Valette, editor, 15th International Conference on the Application and Theory of Petri Nets, volume 815 of Lecture Notes in Computer Science, pages 338-357, Zaragoza, 1994. Springer-Verlag.

13. C.A. Lakos. From Coloured Petri Nets to Object Petri Nets. In G. De Michelis and M. Diaz, editors, 16th International Conference on the Application and Theory of Petri Nets, volume 935 of Lecture Notes in Computer Science, pages 278-297, Torino, Italy, 1995. Springer.

14. C.A. Lakos. State Space Exploration of Object-Based Systems. Technical Report TR05-01, Department of Computer Science, University of Adelaide, April 2005.

15. M. Mäkelä. Maria: Modular Reachability Analyser for Algebraic System Nets. In J. Esparza and C. Lakos, editors, 23rd International Conference on the Application and Theory of Petri Nets, volume 2360 of Lecture Notes in Computer Science, pages 434-444, Adelaide, Australia, 2002. Springer.

16. R. Milner. Elements of Interaction. Communications of the ACM, 36(1):78-89, 1993. ACM.

17. R. Milner. The Flux of Interaction. In J.-M. Colom and M. Koutny, editors, International Conference on the Application and Theory of Petri Nets, volume 2075 of Lecture Notes in Computer Science, pages 19-22, Newcastle, 2001. Springer-Verlag.

18. W. Reisig and G. Rozenberg, editors. Lectures on Petri Nets I: Basic Models, volume 1491 of Lecture Notes in Computer Science. Springer, Berlin, 1998.

19. W. Reisig and G. Rozenberg, editors. Lectures on Petri Nets II: Applications, volume 1492 of Lecture Notes in Computer Science. Springer, Berlin, 1998.

20. R. Valk. Object Petri Nets - Using the Nets-within-Nets Paradigm. In J. Desel, W. Reisig, and G. Rozenberg, editors, Lectures on Concurrency and Petri Nets, volume 3098 of Lecture Notes in Computer Science, pages 819-848. Springer-Verlag, 2004.

21. J. Vitek and G.Castagna. Towards a Calculus of Secure Mobile Computations. In IEEE Workshop on Internet Programming Languages, Chicago, 1998. IEEE. 